\section{Phenylalanin im Blut und Urin}

\author{
W. G. Guder \\ München, Deutschland
}

Synonym(e) Phenylketonurie; PKU; Hyperphenylalaninämie; HPA

Englischer Begriff phenylalanine in blood and urine; phenylketonuria (PKU); hyperphenylalaninemia

Funktion - Pathophysiologie Ursache ist der enzymatische Defekt der Phenylalaninhydroxylase im Genlocus 12q24.1 mit mehr als 400 Mutationen. Dieses Enzym katalysiert die Bildung von $>$ Tyrosin. Mildere Formen, z. B. durch Defekte in der Bildung von Tetrahydrobiopterin, dem Kofaktor des Enzyms, führen zur Hyperphenylalaninämie ohne die gesteigerte Ausscheidung von toxischen Metaboliten. Die Krankheit führt, wenn nicht früh erkannt und durch diätetische Behandlung mit phenylalaninarmer Diät, zu zerebralen Entwicklungsstörungen und Demenz. Die Erkrankung tritt in Deutschland derzeit mit einer Häufigkeit von 1:10.000 Geburten auf.

Untersuchungsmaterial - Entnahmebedingungen Trockenblut auf Filterpapier im Rahmen des Neugeborenenscreenings, die als Probe am 4.-6. Tag nach Geburt gewonnen wird. Urinproben, wie sie früher zum Nachweis der Metaboliten verwendet wurden, sind historisch, da nicht mehr notwendig.

Analytik Der früher durchgeführte Guthrie-Test, der auf dem Wachstum von gehemmten Bakteriensporen von Bacillus subtilis durch vermehrte Phenylalaninkonzentration in der Probe beruhte, ist vollständig durch die beim Neugeborenenscreening angewendete Tandemmassenspektrometrie (vgl. - Massenspektrometrie) ersetzt, die neben der Phenylalaninkonzentration das Phe/Tyr-Verhältnis erfasst.
Konventionelle Einheit $\mathrm{mg} / \mathrm{dL}$.

Internationale Einheit $\mu \mathrm{mol} / \mathrm{L}$.

Umrechnungsfaktor zw. konv. u. int. Einheit 60,5.

Referenzbereich - Erwachsene $>18$ Jahre $35-85 \mu \mathrm{mol} / \mathrm{L}$.

Referenzbereich - Neugeborene $38-137 \mu \mathrm{mol} / \mathrm{L}$.

Referenzbereich - Kinder 2-18 Jahre 26-91 $\mu \mathrm{mol} / \mathrm{L}$. Entscheidungsgrenze beim Screening im Blut: $<242 \mu \mathrm{mol} / \mathrm{L}$ $(<4 \mathrm{mg} / \mathrm{dL})$.

Indikation In Deutschland im Rahmen des Neugeborenenscreenings bei jedem Neugeborenen durchzuführen. Therapieüberwachung bis zum 10. Lebensjahr und vor bzw. in der Schwangerschaft von betroffenen Patientinnen, die erkrankt sind.

Interpretation Bei Erhöhung des Phenylalanins $>250 \mu \mathrm{mol} / \mathrm{L}$ ist auf der Basis des Phe/Tyr-Quotienten Phenylketonurie von eher benigner Hyperphenylalaninämie zu unterscheiden.

Diagnostische Wertigkeit Die Messung des Phenylalanins bei jedem Neugeborenen hat bei konsequenter Durchführung der Therapie zu einem Verschwinden der Krankheit geführt. Die Therapie muss bei schwangeren Betroffenen zum Schutz des Neugeborenen fortgesetzt werden.

\section{Literatur}

Beschluss des Gemeinsamen Bundesausschusses über eine Änderung der Kinder-Richtlinien: Anpassung des erweiterten NeugeborenenScreenings an das Gendiagnostikgesetz (GenDG) (2011) D Ärzteblatt 108:C796-801

Richtlinien des Bundesausschusses der Ärzte und Krankenkassen über die Früherkennung von Krankheiten bei Kindern bis zur Vollendung des 6. Lebensjahres (Kinder-Richtlinien) Bundesanzeiger Nr. 26 vom 21.03.2000 